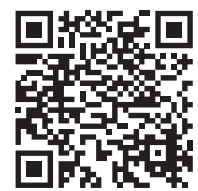

\footnotetext{
Palabras clave:

Ética médica, comunicación de error médico, comunicación médico-paciente, seguridad del paciente.

Keywords: Medical ethics, disclosure of medical errors, provider-patient communication, patient safety.
}

\footnotetext{
* Manager,

Interprofessional Learning and Simulation.

* Fellow, Clinical

Simulation Education and Research

Fellowship.

$\S$ Alumni, Simulation

Center International

Medical Graduate

(IMG) Program, Penn

State Health Milton

S. Hershey Medical

Center. Estados Unidos.
}

Recibido: 09/05/2020

Aceptado: 25/11/2020

doi: $10.35366 / 97903$

\section{Comunicación de errores médicos: un caso de simulación para profesionales de la salud hispanohablantes}

\author{
Medical error disclosure: a simulation case for \\ Spanish-speaking healthcare professionals \\ David Rodgers, * Daniel Enrique Rodríguez-Bauzá,` Miguel Enrique Silva-Rodríguez ${ }^{\S}$
}

El error de medicación es una causa importante de morbilidad y mortalidad en el paciente, pero puede ser un concepto confuso y poco apreciado. En la práctica, un sistema integral que evalúa el daño, la causa raíz y los aspectos psicológicos de los errores es beneficioso para optimizar la comunicación y ayudar a prevenir futuros errores. En este caso de una hora y media de duración, el paciente " $X$ " de 65 años, sexo masculino, con antecedentes de enfermedad de Parkinson, demencia avanzada y diabetes mellitus tipo 2, se presenta al Departamento de Urgencias con empeoramiento progresivo en su estado general, náuseas y vómitos de contenido alimenticio, fiebre, confusión y agresividad, es acompañado por su hija, quien es su representante legal y cuidadora. Es admitido con diagnóstico al ingreso: cetoacidosis diabética, pielonefritis aguda complicada y choque séptico, siendo trasladado a la Unidad de Cuidados Intensivos, con la actuación inmediata del equipo médico se logra estabilidad hemodinámica, reposición hídrica, corrección de la acidosis y de los trastornos electrolíticos, comenzando con tratamiento antibiótico empírico. Durante el quinto día de estadía hospitalaria el residente " $A$ " prescribe inadvertidamente laxantes para el paciente " $X$ ", en vez del paciente "Y". Este error trae como consecuencia un estado de deshidratación moderado secundario a varios episodios de diarrea, requiriendo ajustes en la reposición hídrica. Debido a que el estado mental alterado y la demencia avanzada de este paciente impiden que pueda entender lo que ocurrió, es más apropiado que el equipo médico comunique el error a sus familiares. Los objetivos son el desarrollo de habilidades para la comunicación de errores médicos en un entorno simulado seguro, utilizando el protocolo para la comunicación de errores médicos de R. Buckman. Este caso de simulación clínica permite a los participantes un marco útil para comprender y analizar conflictos éticos, lo que inspirará a tomar decisiones clínicas éticas reflexivas $\mathrm{y}$ tener herramientas para enfrentar estos eventos.

\section{RESUMEN}

\section{ABSTRACT}

Medication error is a major cause of patient morbidity and mortality, but it can be a confusing and under-appreciated concept. Medication error is a major cause of patient morbidity and mortality, but it can be a confusing and under-appreciated concept. In practice, a comprehensive system that assesses damage, root cause, and psychological aspects of errors is beneficial in modifying communication and helping to prevent future errors. In this 1.5-hour simulation scenario, a 65-year-old male patient " $X$ " with history of Parkinson's disease, advanced dementia and type 2 diabetes mellitus presented to the Emergency Department with progressive worsening in his general condition, nausea and vomiting of food content, fever, confusion and aggressiveness accompanied by his daughter who is his legal representative and caregiver. He is admitted with a diagnosis on admission: diabetic ketoacidosis, complicated acute pyelonephritis, and septic shock, being transferred to the Intensive Care Unit, with the immediate action of the medical team, hemodynamic stability, fluid replacement, correction of acidosis and electrolyte disorders are archived, starting also broad spectrum antibiotic treatment. During the 5 th day of hospital stay, resident " $A$ " inadvertently prescribed laxatives for patient " $X$ " thinking he was writing the order for patient " $Y$ ". This error results in moderate dehydration after several episodes of diarrhea, requiring adjustments in fluid replacement. Because this patient's altered mental state and advanced dementia prevent the patient from understanding of what occurred, it is more appropriate for the medical team to disclose the error to his daughter. The objectives are to develop skills for medical errors disclosure in a safe simulated environment, using Buckman's protocol for communication of medical errors. This clinical simulation case allows participants a useful framework for understanding and analyzing ethical conflicts, inspiring them to make thoughtful ethical clinical decisions and have tools to deal with these events.
Citar como: Rodgers D, Rodríguez-Bauzá DE, Silva-Rodríguez ME. Comunicación de errores médicos: un caso de simulación para profesionales de la salud hispanohablantes. Simulación Clínica. 2020; 2 (3): 140-146. https://dx.doi.org/10.35366/97903 


\section{INTRODUCCIÓN}

$E^{\prime}$ error médico es una causa importante de morbilidad y mortalidad. Desde el informe histórico del Instituto de Medicina de los Estados Unidos (OIM) "Errar es humano: construir un sistema de salud más seguro", se ha tenido un reconocimiento mayor del impacto del error médico. ${ }^{1}$ Junto con este reconocimiento ha crecido la importancia de "atención centrada en el paciente". Un elemento clave que respalda la atención centrada en el paciente es la necesidad de honestidad con éste, incluida la necesidad de revelar errores médicos. ${ }^{2}$ La primera exposición de muchos médicos a errores ocurre en el entrenamiento. ${ }^{3,4}$ En un estudio reciente $79 \%$ de los estudiantes de medicina de cuarto año y $98 \%$ de los residentes reportaron experiencia con errores médicos. ${ }^{5}$ La mayoría creía que estos errores deberían comunicarse a los pacientes. ${ }^{5,6}$ A pesar de sus creencias, muchos residentes informaron que cuando ocurre un error pueden no revelarlo $\mathrm{o}$, si lo hacen, su explicación puede no incluir todos los detalles relevantes. ${ }^{3-6}$ Además, abundantes encuentros de médicos en formación con errores médicos carecen de una disculpa explícita al paciente, ${ }^{5}$ que muchos ven ahora como un elemento crucial en la comunicación de errores médicos y malas noticias a pacientes y familiares.

Incluso en culturas y países que han aceptado la necesidad de revelar errores médicos, existen barreras y desafíos que incluyen reacciones negativas tanto para el proveedor de atención médica como para el paciente, sentimientos como el miedo a la culpa y el potencial de litigios, la falta de habilidad para revelar errores y la ansiedad. 2,7,8 Para contrarrestar esto, la comunicación de errores médicos ahora se incluye en la educación de residencia en los EUA. ${ }^{9}$

La divulgación de errores médicos en América Latina tiene barreras adicionales, ya que existen muy pocas regulaciones para identificar o rastrear errores médicos; ${ }^{10}$ sin embargo, cada vez se reconoce más el informe de estos errores. ${ }^{11,12}$ Algunas culturas han sido más lentas en responder por la necesidad de "decir la verdad" en la divulgación del error médico como parte de la atención centrada en el paciente. ${ }^{13}$ Esto, combinado con las opiniones del médico como una figura de autoridad, hace que la divulgación de errores médicos sea un problema en la cultura latina. ${ }^{14}$

Uno de los modelos de comunicación más destacados para la comunicación de errores médicos es el modelo CONES (siglas en inglés), desarrollado por Robert Buckman. ${ }^{15}$ Este protocolo presenta cinco pasos:

- Paso 1. Contexto (Context): lugar privado donde puedan sentarse médico y paciente o familiares.

Esta etapa de preparación implica tener conocimiento del paciente, de lo ocurrido, así como una preparación emocional.

- Paso 2. Apertura (Opening remark): declaración inicial que define el objetivo del encuentro y demuestra que usted está preparado para discutir una situación importante.

- Paso 3. Narrativa (Narrative): ofrecer un recuento cronológico de los eventos y de responder a las interrogantes y sentimientos que emergen.

- Paso 4. Manejo emocional (Emotions): identificar las emociones, determinar el motivo de la emoción y responder empáticamente.

- Paso 5. Plan de acción y resumen (Strategy and Summary): explicar un plan que manifieste que el profesional y el paciente persiguen un mismo objetivo, así como estrategias institucionales para la prevención de futuros errores de esta índole.

El objetivo de este caso de simulación es presentar una aplicación contextual para la comunicación de errores médicos a un miembro de la familia del paciente. Se divide en tres etapas: prebriefing (10 minutos), escenario de simulación (20-30 minutos) y debriefing (40 minutos). Los objetivos específicos son:

1. Revisar el incidente (error médico).

2. Practicar la comunicación de un error médico al paciente y su familia en un entorno simulado seguro, utilizando el protocolo de Buckman para la comunicación de errores médicos.

3. Demostrar una comunicación efectiva en línea con el protocolo de cinco pasos.

4. Discutir la evidencia de las expectativas médico-paciente en la comunicación de errores médicos a pacientes y familiares durante la sesión informativa.

5. Explorar los riesgos y beneficios de informar errores médicos durante la sesión informativa.

\section{PRESENTACIÓN DEL CASO}

Paciente " $X$ ", de 65 años de edad, sexo masculino con antecedentes de enfermedad de Parkinson, demencia avanzada y diabetes mellitus tipo 2 . Acude al Departamento de Urgencias relatando 
que 14 días antes había iniciado un cuadro de disuria que lo llevó a que su médico de cabecera le diagnosticara infección urinaria y le prescribiera un ciclo corto de antibiótico. Siete días después, y una vez finalizado el tratamiento, vuelve a acudir con su médico por iniciar un dolor en región lumbar izquierda de naturaleza constante y exacerbado con los movimientos, para el cual se le prescribieron relajantes musculares. A los cuatro días de haber iniciado el nuevo tratamiento sufre un empeoramiento progresivo en su estado general acompañado de náuseas y vómitos de contenido alimenticio, con escalofríos, fiebre, anorexia, confusión y agresividad, por lo que el paciente acude a urgencias acompañando por su hija, quien es su representante legal y cuidadora.

Al examen físico se constata palidez cutáneomucosa, toma del estado general, dolor localizado en fosa iliaca derecha (FID), sin signos de irritación peritoneal, prueba de puño percusión positiva bilateral, TA-60/40, pulso-115'.

Resultados de laboratorio clínico e imagenología al ingreso:

1. En el examen de orina se aprecia: sedimento: 6-8 hematíes/campo, 20-25 leucos/campo, algunos gérmenes. $\mathrm{Na} 2+$ en orina $108 \mathrm{mEq} / \mathrm{L}$ y K + de $14 \mathrm{mEq} / \mathrm{L}$. Glucosuria y cetonuria: +.

2. Hemograma: Hto 47\%, leucocitos 22,000/ $\mathrm{mm}^{3}$, plaquetas 183,000. Coagulación: TC 8 min, TPTA 5 seg. Tiempo protrombina 3 seg.

3. Bioquímica: urea $125 \mathrm{mg} / \mathrm{dL}$, creatinina 3.5 $\mathrm{mg} / \mathrm{dL}, \mathrm{K}+7.6 \mathrm{mEq} / \mathrm{L}, \mathrm{Na} 2+108 \mathrm{mEq} / \mathrm{L}$, glucosa $1,430 \mathrm{mg} / \mathrm{dL}$.

4. Gasometría arterial: $\mathrm{pH}$ 6.95, bicarbonato $\mathrm{HCO} 318 \mathrm{mEq} / \mathrm{L}$.

5. Ecografía abdominal al ingreso: ambos riñones con pobre delimitación cortico-medular, no litiasis o alteraciones anatómicas.

Se tomaron hemocultivos y cultivo de orina (urocultivo) al ingreso.

Es admitido con diagnóstico al ingreso: cetoacidosis diabética, pielonefritis aguda complicada y choque séptico y trasladado a la Unidad de Cuidados Intensivos, con la actuación inmediata del equipo médico se logra estabilidad hemodinámica, reposición hídrica, corrección de la acidosis y de los trastornos electrolíticos comenzando con tratamiento antibiótico empírico (piperacilinatazobactam [Zosyn] 3.375 g IV cada seis horas).

Se recibe el resultado de los hemocultivos y del cultivo de muestras tomada durante el ingreso, coincidiendo ambos en el crecimiento de un $E$. coli sensible a cefalosporinas de tercera generación, betalactámicos más inhibidores de las betalactamasas, aminoglucósidos, quinolonas y carbapenémicos.

El paciente evolucionó favorablemente, con buena recuperación del estado de choque, afebril, con parámetros inflamatorios dentro de rangos normales y sin deterioro de la función renal. Durante el séptimo día de estadía hospitalaria, el residente " $\mathrm{A}$ " recetó inadvertidamente laxantes para el paciente " $X$ " pensando que estaba escribiendo la orden para el paciente " $Y$ ". El paciente " $X$ " ha presentado varios episodios de diarrea durante el turno de la noche. Este error trae como consecuencia un estado de deshidratación moderado que requirió ajustes en la reposición hídrica. Debido a que el estado mental alterado y la demencia avanzada de este paciente impiden que él pueda entender lo que ocurrió, es más apropiado que el equipo médico comunique el error a sus familiares. Con el objetivo de comunicar el error médico, una reunión con los familiares ha sido planificada para hoy.

\section{Implementación}

Lugar: privacidad (habitación con iluminación y ventilación apropiada), asientos para todos y pañuelos de papel.

Ubicación temporal-espacial: reunión con familiares para comunicarles el error médico.

El caso de simulación utiliza una actriz para desempeñar el papel de la hija que es el representante legal del paciente. Se informará a este actor sobre el escenario y se le proporcionará instrucciones sobre cómo responder verbal y emocionalmente:

- $\mathrm{Al}$ inicio:

1. Emociones: expresión facial de preocupación.

2. Diálogo (cuando entre el equipo médico): "¿Qué le pasó a mi padre?, ayer lo vi y estaba mejorando" iOooh! ¿Qué está pasando doctores, le ocurrió algo a mi padre?

- Cuando el error médico es comunicado:

1. Emociones: rabia, interrumpe a los médicos varias veces, camina alrededor de la habitación, demuestra enojo. 
2. Diálogo: "Esto es ridículo e inadmisible". "No entiendo cómo situaciones como ésta pasen, yo perdí a mi hijo hace cinco años por negligencia médica. Y aún siguen pasando situaciones como ésta". (Cúbrete la cara y finge llorar).

"Esto no se va quedar así, pudieron haber matado a mi padre, ¿qué tipo de hospital es éste?".

Si se menciona laxante: "¿Qué tipo de medicamento es eso -laxante-?"

Los participantes tendrán prebriefing con la descripción del caso y objetivos educativos (cinco minutos).

El equipo médico se reúne, asigna tareas y roles, esta etapa incluye la preparación del profesional, que no sólo implica tener conocimiento del paciente y de lo ocurrido, sino también una preparación emocional. Aclarar los objetivos y decidir la terminología general que se utilizará. Quién debería dar la noticia, y qué personal debe estar presente (uno de los participantes asumirá el rol del residente que cometió el error médico).

El equipo entra a la habitación donde el familiar está esperando, éstos se presentan (nombre completo y rol en el hospital) y mantienen contacto visual. Los participantes deben hacer uso del protocolo para la comunicación de errores médicos de R. Buckman, ${ }^{15}$ incluyendo una disculpa por lo ocurrido. Este protocolo fue traducido al español con el acrónimo CANEP correspondientes a: Contexto (Context), Apertura (Opening remark), Narrativa (Narrative), Emociones (Emotions) y Plan y Resumen (Strategy and Summary). ${ }^{16}$

El diálogo debe incluir elementos que idealmente incluyan: 1) notificación de que un error ha ocurrido, 2) descripción de lo que sucedió, 3) una sincera disculpa, 4) aceptación de responsabilidad, 5) descripción de los siguientes pasos a tomarse para mitigar el daño y corregir la situación, y 6) asegurar una investigación para prevenir la recurrencia. ${ }^{3,17,18}$ Respondiendo con empatía a la respuesta emocional de familiares, legitimándolos.

\section{DISCUSIÓN}

Los errores médicos son un grave problema de salud pública y una de las principales causas de muerte en los Estados Unidos. Es un problema difícil, ya que es complicado descubrir una causa constante de errores, e incluso si se encuentra, proporcionar una solución viable consistente que minimice las posibilidades de un evento recurrente. $\mathrm{Al}$ reconocer que ocurren eventos adversos, aprender de ellos y trabajar para prevenirlos puede mejorar la seguridad del paciente. ${ }^{19}$ Parte de la solución es mantener una cultura que trabaje para reconocer los desafíos de seguridad e implementar soluciones viables en lugar de albergar una cultura de culpa, vergüenza y castigo. Las organizaciones de atención médica deben establecer una cultura de seguridad que se centre en la mejora del sistema al ver los errores médicos como desafíos que deben superarse. Todas las personas del equipo de atención médica deben desempeñar un papel en hacer que la provisión de la atención médica sea más segura para los pacientes y los trabajadores de la salud. ${ }^{20}$ La mayor parte de la atención médica se realiza ahora por equipos multidisciplinarios y los errores pueden constituir el final de una secuencia de eventos que pueden involucrar a muchos "contribuyentes". Los errores de medicación juegan un papel importante en la morbilidad y mortalidad del paciente, particularmente en el entorno hospitalario. Estos errores pueden ser causados por profesionales de la salud (por ejemplo, errores al ingresar el medicamento, no renovar el medicamento), factores relacionados con el medicamento (nombres similares, ventana terapéutica estrecha) y factores relacionados con los pacientes (insuficiencia renal, polifarmacia).

Partiendo de lo antes expuesto el debriefing (40 minutos) debe ser una experiencia educativa en la que se profundice terminología y definiciones, incidencia, factores de riesgo, estrategias de prevención y comunicación de errores médicos, así como las consecuencias legales. Se puede encontrar una variedad de estrategias para la realización del debriefing en la literatura; sin embargo, la herramienta Gather-Analyze-Summarize (GAS), ${ }^{21}$ es probablemente una de las más fáciles de entender. Por lo que la hemos traducido al español con las siglas RAR (recopilar, analizar y resumir), e incluye preguntas y puntos de discusión que recomendamos durante el debriefing (Apéndice A). Sugerimos que el facilitador fomente la reflexión y participación de todos los educandos a lo largo del debriefing.

\section{CONCLUSIONES}

Los autores reconocen que existen obstáculos sustanciales, incluidas las expectativas poco realistas de infalibilidad del médico, la falta de 
capacitación sobre la comunicación de errores y el temor a una mayor exposición a negligencia, que pueden obstruir la comunicación a los pacientes de errores médicos. Para superar estos obstáculos, es importante que las instituciones de atención médica desarrollen e implementen políticas y procedimientos para identificar y responder a errores médicos, incluyendo sistemas y procedimientos para revelar errores significativos a los pacientes, lo que influirá positivamente en mejoras en la calidad de los servicios médicos. Los educadores deben desarrollar y proporcionar instrucciones específicas a los residentes en todos los niveles sobre cómo identificar y prevenir errores médicos y cómo comunicarlos de manera veraz y sensible a los pacientes o sus familiares.

La simulación clínica es una de las herramientas más utilizadas actualmente en la formación de profesionales de la salud y constituye una herramienta para capacitar y promover un ambiente de seguridad al paciente. Este caso de simulación es un recurso autónomo con todas las instrucciones necesarias para proporcionar una experiencia educativa exitosa, proporciona el desarrollo de habilidades de comunicación de errores médicos, herramientas para superar barreras, reflejar experiencias, describir cómo se resolverán desafíos e inspirar a tomar decisiones clínicas en concordancia con normas éticas establecidas. Se puede usar de forma aislada o en combinación con otros recursos publicados. Se necesitarán investigaciones en el futuro para evaluar la repercusión y/o resultados de esta actividad educativa en la atención médica. Sin embargo, este caso de simulación demuestra potencial como una valiosa herramienta educativa para los médicos residentes.

\section{AGRADECIMIENTOS}

Agradecemos al Centro de Simulación Clínica de Penn State Health Milton S. Hershey Medical Center por fomentar su misión de mejorar la seguridad en el paciente con programas efectivos que promuevan y mejoren las habilidades de los profesionales, la competencia clínica, el trabajo en equipo y la colaboración interdisciplinaria; avanzando el campo de la simulación en salud, promoviendo investigación innovadora sobre la teoría, la práctica y la tecnología de simulación para el beneficio de profesionales de la salud hispanohablantes.

\section{REFERENCIAS}

1. Kohn LT, Corrigan JM, Donaldson MS, eds. Committee on Quality of Health Care in America, Institute of Medicine. Washington, DC, USA: National Academies Press; 1999.

2. Yürür G, Valdez KPR. Disclosure of medical errors: a literature review and the situation in Turkey. Reports in Advances of Physical Sciences. 2018; 2 (1): 1850003. doi: 10.1142/S2424942418500032.

3. Wu AW, Folkman S, McPhee SJ, Lo B. Do house officers learn from their mistakes? JAMA. 1991; 265 (16): 2089-2094.

4. Kaldjian LC, Jones EW, Wu BJ, Forman-Hoffman VL, Levi BH, Rosenthal GE. Disclosing medical errors to patients: Attitudes and practices of physicians and trainees. J Gen Intern Med. 2007; 22: 988-996.

5. White AA, Gallagher TH, Krauss MJ, et al. The attitudes and experiences of trainees regarding disclosing medical errors to patients. Acad Med. 2008; 83: 250-256.

6. White AA, Bell SK, Krauss MJ, et al. How trainees would disclose medical errors: Educational implications for training programmers. Med Educ. 2011; 45: 372-380.

7. Sattar R, Johnson J, Lawton R. The views and experiences of patients and health-care professionals on the disclosure of adverse events: a systematic review and qualitative meta-ethnographic synthesis. Health Expect. 2020. doi: 10.1111/hex.13029. [Epub ahead of print]

8. Koller D, Rummens A, Le Pouesard M, Espin S, Friedman J, Coffey $M$, et al. Patient disclosure of medical errors in paediatrics: A systematic literature review. Paediatr Child Health. 2016; 21 (4): e32-e38.

9. American Council on Graduate Medical Education (ACGME) Common Program requirements (Residency). 2019. Available in: https://www.acgme.org/Portals/0/ PFAssets/ProgramRequirements/CPRResidency2019.pdf

10. Alvarado-Guevara AT, Flores-Sandí G. Errores médicos. Acta Méd Costarric. 2009; 51 (1): 16-23.

11. Aranaz-Andrés JM, Aibar-Remón C, Limón-Ramírez R, Amarilla A, Restrepo FR, Urroz O, et al. Prevalence of adverse events in the hospitals of five Latin American countries: results of the 'Iberoamerican Study of Adverse Events' (IBEAS). BMJ Qual Saf. 2011; 20 (12): 1043-1051. doi: 10.1136/bmjqs.2011.051284.

12. Shanks L, Bil K, Fernhout J. Learning without borders: a review of the implementation of medical error reporting in Médecins sans Frontières. PLoS One. 2015; 10 (9): e0137158. doi: 10.1371/journal. pone. 0137158 .

13. Macklin R. The doctor-patient relationship in different cultures. In: Macklin R. Against relativism: cultural diversity and the search of ethical universals in medicine. Oxford University Press, Inc.; 1999. pp. 86-107.

14. Flores G. Culture and the patient-physician relationship: achieving cultural competency in health care. J Pediatr. 2000; 136 (1): 14-23.

15. Buckman R. Difficult conversations in medicine 2010. EE.UU: The John Hopkins University press; 2010.

16. Bascuñán ML, Arriagada AM. Comunicación de errores médicos a pacientes y familiares: interrogantes y herramientas. Rev Med Chile. 2016; 144: 1185-1190. 
17. Gallagher $T H$, Waterman $A D$, Ebers $A G$, Fraser VJ, Levinson W. Patients' and physicians' attitudes regarding the disclosure of medical errors. JAMA. 2003; 289 (8): 1001-1007.

18. Vincent C, Young M, Phillips A. Why do people sue doctors? A study of patients and relatives taking legal action. Lancet. 1994; 343 (8913): 1609-1613.

19. Oyebode F. Clinical errors and medical negligence. Med Princ Pract. 2013; 22 (4): 323-333.

20. Helo S, Moulton CE. Complications: acknowledging, managing, and coping with human error. Transl Androl Urol. 2017; 6 (4): 773-782.
21. PhrampusP,O'Donnell].Debriefingusingastructured and supported approach. In: The comprehensive textbook of healthcare simulation. New York: Springer; 2014. pp. 73-85.

\section{Correspondencia:}

Daniel Enrique Rodríguez-Bauzá, MD.

500 University Drive

Mail Code H128, Room C2630D

Hershey, PA 17033.

E-mail: drodriguezbauza@

pennstatehealth.psu.edu 
Apéndice A.

\begin{tabular}{|c|c|c|c|c|}
\hline & Objetivos & Acciones & Ejemplos de preguntas & Tiempo \\
\hline $\begin{array}{c}\text { G } \\
\text { Recopilar }\end{array}$ & $\begin{array}{l}\text { I. Escuche a los participantes } \\
\text { para comprender cómo se } \\
\text { sienten } \\
\text { II. Identifica lo que sucedió } \\
\text { durante el escenario (etapa de } \\
\text { preparación y el encuentro con } \\
\text { los familiares) }\end{array}$ & $\begin{array}{l}\text { - Obtén el estado emocional de } \\
\text { los participantes } \\
\text { - Solicita narrativa del líder del } \\
\text { equipo } \\
\text { - Solicita la aclaración por parte } \\
\text { del equipo de sus acciones }\end{array}$ & $\begin{array}{l}\text { ¿Podrías describir lo ocurrido desde tu perspectiva? } \\
\text { ¿Cómo fue el proceso de asignación de tareas? } \\
\text { ¿Puedes hablarme acerca de la etapa de preparación } \\
\text { del profesional? } \\
\text { ¿Tuvieron alguna preparación emocional? } \\
\text { ¿Cuál fue la reacción emocional de los familiares? } \\
\text { ¿Cómo manejaron estas relaciones emocionales? } \\
\text { También incluye tus observaciones: } \\
\text { Noté que... } \\
\text { Observé que ... }\end{array}$ & $\begin{array}{c}10 \\
\text { minutos }\end{array}$ \\
\hline $\begin{array}{c}\text { A } \\
\text { Analizar }\end{array}$ & $\begin{array}{l}\text { I. Asegure un enfoque continuo } \\
\text { en los objetivos de la sesión } \\
\text { II. Facilite la reflexión y el } \\
\text { análisis de los participantes } \\
\text { sobre sus acciones individuales } \\
\text { III. Facilitar la reflexión y el } \\
\text { análisis del equipo sobre los } \\
\text { problemas del sistema }\end{array}$ & $\begin{array}{l}\text { - Revisión exacta de los eventos } \\
\text { - Ayude a los participantes } \\
\text { a reflexionar sobre su } \\
\text { desempeño y la aplicación de } \\
\text { principios éticos } \\
\text { - Infórmales tus observaciones } \\
\text { (pasos correctos / incorrectos) } \\
\text { - Haga una serie de preguntas } \\
\text { para revelar el proceso } \\
\text { de pensamiento de los } \\
\text { alumnos y relacionarse } \\
\text { con las responsabilidades } \\
\text { interprofesionales en la } \\
\text { implementación de buenas } \\
\text { prácticas médicas } \\
\text { - Refuerza lo positivo y } \\
\text { considere acciones para } \\
\text { cambiar su comportamiento }\end{array}$ & $\begin{array}{l}\text { Me di cuenta... } \\
\text { Cuéntame más sobre ... (e.g. Los elementos que } \\
\text { incluyeron en el diálogo con el paciente. También los } \\
\text { elementos que deben ser idealmente incluidos) } \\
\text { ¿Cómo te sentiste acerca de ... (e.g. Uso del } \\
\text { protocolo para la comunicación de errores traducido } \\
\text { al español con el acrónimo CANEP) } \\
\text { ¿Qué estabas pensando cuando ...? } \\
\text { Entiendo; sin embargo, cuéntame sobre el aspecto } \\
\text { "X" del escenario } \\
\text { ¿Podrían comentarme acerca de alguna experiencia } \\
\text { en la identificación de factores de riesgo, estrategias } \\
\text { de prevención y comunicación de errores médicos? } \\
\text { ¿Qué aspectos salieron bien? } \\
\text { ¿Qué aspectos necesitan mejorar? } \\
\text { Informe sus observaciones e integre el Código de } \\
\text { Ética Médica de su institución }\end{array}$ & $\begin{array}{c}20 \\
\text { minutos }\end{array}$ \\
\hline $\begin{array}{c}\text { S } \\
\text { Resumir }\end{array}$ & $\begin{array}{l}\text { I. Facilite la identificación y } \\
\text { revisión de las lecciones } \\
\text { aprendidas }\end{array}$ & $\begin{array}{l}\text { - Identifique aspectos positivos } \\
\text { de la simulación } \\
\text { - Resuma los comentarios o } \\
\text { discusiones } \\
\text { - Ayude a los participantes a } \\
\text { identificar los puntos para } \\
\text { llevar a la atención médica }\end{array}$ & $\begin{array}{l}\text { Mirando nuestros objetivos originales, } \\
\text { ¿qué hemos aprendido? } \\
\text { ¿Qué harás de manera diferente con base en esta } \\
\text { experiencia? } \\
\text { ¿Cómo se traducirá esta experiencia a tu práctica } \\
\text { médica futura? }\end{array}$ & $\begin{array}{c}10 \\
\text { minutos }\end{array}$ \\
\hline
\end{tabular}

